INPLASY

PROTOCOL

To cite: Yang et al. Meta analysis of distribution frequency of TCM syndromes in depression. Inplasy protocol 202140052. doi:

10.37766/inplasy2021.4.0052

Received: 08 April 2021

Published: 09 April 2021

Corresponding author:

Xuan Zhou

xuetcm@gmail.com

Author Affiliation:

Jinan University

Support: National Natural

Science Found.

Review Stage at time of this submission: Data analysis.

Conflicts of interest:

None declared.

\section{Meta analysis of distribution frequency of TCM syndromes in depression}

Yang, N1; Gan, H2; Wang, Y3; Ma, Q4; Zhou, X5.

Review question / Objective: Understand the distribution and characteristics of TCM syndromes of depression, and provide references for clinical syndrome differentiation, treatment and related research.

Condition being studied: Depression is a common mental disorder, and its prevalence is increasing year by year. It is predicted that by 2030 , depression will become one of the three main causes of disease burden. Years of scientific research and clinical practice have shown that traditional Chinese medicine prescriptions are one of the effective methods for the treatment of depression, and syndrome research is an important breakthrough to improve the level of diagnosis and treatment of traditional Chinese medicine. Understanding the distribution and characteristics of TCM syndromes of depression will help to better Formulate a TCM treatment plan for depression well.

INPLASY registration number: This protocol was registered with the International Platform of Registered Systematic Review and Meta-Analysis Protocols (INPLASY) on 09 April 2021 and was last updated on 09 April 2021 (registration number INPLASY202140052).

\section{INTRODUCTION}

Review question / Objective: Understand the distribution and characteristics of TCM syndromes of depression, and provide references for clinical syndrome differentiation, treatment and related research.

Rationale: Following the evidence-based medicine method, this study conducted a meta-analysis of all published literature data of TCM syndrome-type studies of depression in China that meet certain standards, in order to further clarify the distribution of TCM syndromes of depression.

Condition being studied: Depression is a common mental disorder, and its prevalence is increasing year by year. It is predicted that by 2030, depression will 
become one of the three main causes of disease burden. Years of scientific research and clinical practice have shown that traditional Chinese medicine prescriptions are one of the effective methods for the treatment of depression, and syndrome research is an important breakthrough to improve the level of diagnosis and treatment of traditional Chinese medicine. Understanding the distribution and characteristics of TCM syndromes of depression will help to better Formulate a TCM treatment plan for depression well.

\section{METHODS}

Search strategy: Computer searches of CNKI, WanFang, VIP, CBM, PubMed, Embase and Web of Science databases to collect research on the distribution of TCM syndromes of depression in China. The search time limit is from January 1, 2001 to March 1, 2021. Chinese search terms include: depression, syndrome differentiation, rules of syndrome and treatment, etc.; English search terms include: depression, major depressive disorder, MDD, syndrome, traditional Chinese medicine syndrome, TCM syndrome, etc. The search strategy is determined after multiple pre-searches and adjusted according to different databases.

\section{Participant or population: Mainland Chinese population.}

Intervention: TCM syndrome differentiation or treatment based on syndrome differentiation.

Comparator: The distribution frequency of the five TCM syndromes of depression (Liver stagnation and qi stagnation syndrome, liver depression and spleen deficiency syndrome, liver depression and phlegm block syndrome, heart and spleen deficiency syndrome, and disharmony between heart and kidney syndrome) is unclear.

Study designs to be included: Crosssectional studies.
Eligibility criteria: Inclusion criteria: (1) Meet the CCMD-3 diagnostic criteria for depression; (2) Meet the "Syndrome Differentiation Criteria for Bipolar Disorder Syndrome of Integrated Traditional Chinese and Western Medicine"; (3) Papers involving research on the distribution frequency of TCM syndromes in depression. Exclusion criteria: (1) Repeatedly published research; (2) Documents in languages other than Chinese and English; (3) Secondary depression such as post-stroke depression and other non-primary depression; (4) Theoretical discussion, Literature research, biological basic research and other nonclinical research; (5) Only research a certain TCM syndrome of depression. Inclusion criteria: (1) Meet the diagnostic criteria for depression in the third edition (CCMD-3) of the "Chinese Mental Illness Classification and Diagnosis Standards" revised in 2001 by the Chinese Association of Psychiatry; The Standards for Syndrome Differentiation of Bipolar Depression Syndrome of Integrated Traditional Chinese and Western Medicine formulated by the professional committee in 1991; (3) Journal papers, conference papers, and dissertations related to the research on the distribution frequency of TCM syndromes in depression. Exclusion criteria: (1) Repeated studies (if two or more articles are from the same study, choose the one with the most complete data and the most detailed report); (2) Documents in languages other than Chinese and English; (3) Follow-up Non-primary depression such as post-stroke depression; (4) Non-clinical research such as theoretical discussion, literature research, and basic biological research; (5) Only a certain TCM syndrome of depression is studied, The five types of TCM syndromes in "Bipolar-Stagnation Syndrome of Integrated Traditional Chinese and Western Medicine Syndrome Differentiation Standards" include the syndrome of stagnation of liver and qi, syndrome of stagnation of liver and spleen, syndrome of stagnation of liver and phlegm, syndrome of deficiency of both heart and spleen, and syndrome of heart and kidney failure Fully incorporated papers. 
Information sources: Mainly electronic resources (including CNKI, WanFang, VIP, CBM, PubMed, Embase and Web of Science databases).

Main outcome(s): The distribution frequency of five TCM syndromes in depression: liver stagnation and qi stagnation syndrome, liver stagnation and spleen deficiency syndrome, liver depression and phlegm obstruction syndrome, heart and spleen deficiency syndrome, and disharmony between heart and kidney syndrome.

Data management: Two researchers independently screened the literature according to the inclusion and exclusion criteria, extracted data, and crosschecked. In case of differences, they are resolved through discussion and negotiation.

Quality assessment / Risk of bias analysis: The Agency for Healthcare Research and Quality (AHRQ) quality standards for crosssectional studies were used to evaluate the risk of bias in the included studies. There are a total of 11 items in the AHRQ list. Answers with "yes", "no" and "do not know" are assigned points. "Yes" gets 1 point, and "no" and "do not know" get 0 points. The higher the total score, the higher the quality of the literature. Among them, $0-3$ is divided into low quality, 4-7 is divided into medium quality, and $8-11$ is divided into high quality. Two investigators independently evaluated the risk of bias in the included studies, and cross-checked the results.

Strategy of data synthesis: Meta-analysis was performed using $R \mathbf{4 . 0 3}$ meta package. Firstly, the heterogeneity among the included research results was evaluated by $Q$ test and 12 statistical value. If there is no statistical heterogeneity between the results of each study ( $P>0.1$ and $I 2<50 \%$ ), the fixed effects model is used for Meta analysis; if there is statistical heterogeneity between the results of each study, the random effects model is used for Meta analysis Analyze $(a=0.05)$, and use subgroup analysis or sensitivity analysis to further analyze the source of heterogeneity. The funnel chart was used to evaluate publication bias.

Subgroup analysis: Use subgroup analysis to further analyze the source of heterogeneity if necessary: total number of cases, quality evaluation score.

Sensitivity analysis: Perform sensitivity analysis by eliminating individual studies one by one.

Language: Chinese or English.

Country(ies) involved: China.

Keywords: Depression, major depressive disorder, traditional Chinese medicine syndrome, TCM syndrome, meta analysis.

Contributions of each author:

Author 1 - Nating Yang - The author collected research, extracted data and drafted the manuscript.

Email: yang_nating@163.com

Author 2 - Hua Gan - The author collected research, extracted data and modified the manuscript.

Email: jasmine623223@stu2018.jnu.edu.cn

Author 3 - Yaping Wang - The author provided comments on modifying the article.

Email: ypwang30@163.com

Author 4 - Qingyu Ma - The author provided comments on modifying the article.

Email: tmaqingyu@jnu.edu.cn

Author 5 - Xuan Zhou - The author provided statistical expertise, feedback and approved the final manuscript.

Email: xuetcm@gmail.com 